to respond to illumination from the accretion disk, which, in effect, measures the distance between the disk and the surrounding gas ${ }^{6}$. In a similar way to how bats use echolocation to map out a dark cave, astronomers measure light echoes to map out the hot gas around black holes.

The GRAVITY Collaboration has ushered in an alternative technique that spatially resolves the motion of such gas using the GRAVITY instrument in Chile ${ }^{7}$. This instrument is an interferometer that combines the light from four near-infrared telescopes that are 8 metres in diameter to produce a virtual 'super telescope' that is $130 \mathrm{~m}$ in diameter. Because the spatial resolution of a telescope depends on its size, the use of the GRAVITY instrument is a giant step in imaging capability. The collaboration measured the offset in phase between the direct emission of light from 3C 273 and the light from the broad-line region to spatially resolve the motion of this gas in a distant quasar for the first time.

The team observed a velocity gradient in the gas on size scales of 10 microarcseconds - an achievement that is comparable to seeing a coin on the Moon from Earth. The researchers found that the motion of this gas is perpendicular to the known large-scale jet (a beam of charged particles) projected from 3C 273 (Fig. 1). The results suggest that the gas is in the form of a thick ring with a radius of 0.12 parsecs, rotating around a black hole that has a mass 300 million times that of the Sun. These findings support previous estimates from reverberation mapping of 3C 273 that indicated a similar black-hole mass and gravitationally bound gas at a distance of 0.08-0.34 parsecs from the black hole ${ }^{8,9}$.

For astronomers, the excitement about the current work is not because the results have fundamentally changed our understanding of quasars, but rather because this impressive technological advance enables an independent cross-check of optical-reverberation mapping - the most widely used method for determining the structure of gas around supermassive black holes. Optical reverberation has been measured in roughly 60 quasars $^{10}$, and the inferred properties of the gas strongly correlate with the luminosity of the quasar and the mass of the central black hole.

These correlations have been applied to large samples that comprise thousands of quasars. They have thereby informed our understanding of far-reaching aspects of astronomy, from the co-evolution of black holes and galaxies over cosmic time to the rate at which the expansion of the Universe is accelerating. Having an independent cross-check from spatially resolved interferometric observations, as reported by the GRAVITY Collaboration, is valuable for confirming several key findings in astrophysics that rely on the robustness of reverberation-mapping results.

It is important to keep in mind that the results presented in the paper are based on one particular quasar. The GRAVITY Collaboration observed 3C 273 because it was the best target for optical interferometry. However, the quasar is by no means the best target for reverberation mapping, which makes it difficult to compare the results from these two methods critically.

Going forward, the GRAVITY instrument should be capable of spatially resolving the dynamics and orientations of the broadline region in about ten other quasars ${ }^{11}$. To best corroborate or dispute sizes and structures inferred from reverberation mapping, coordinated campaigns on the same quasars using two independent techniques must be carried out. The GRAVITY instrument is at the beginning of its scientific operations, and these early technical achievements bode well for future investigations that peer deeper into the hearts of quasars.
Erin Kara is in the Department of Astronomy, University of Maryland, Maryland 20742, USA. e-mail:ekara@astro.umd.edu

1. Edge, D. O., Shakeshaft, J. R., McAdam, W. B., Baldwin, J. E. \& Archer, S. Mem. R. Astron. Soc. 68, 37-60 (1959).

2. Hazard, C., Mackey, M. B. \& Shimmins, A. J. Nature 197, 1037-1039 (1963).

3. Schmidt, M. Nature 197, 1040 (1963)

4. GRAVITY Collaboration. Nature $563,657-660$ (2018).

5. Blandford, R. D. \& McKee, C. F. Astrophys. J. 255, 419-439 (1982).

6. Peterson, B. M. Publ. Astron. Soc. Pacif. 105 247-268 (1993)

7. GRAVITY Collaboration. Astron. Astrophys. 602, A94 (2017).

8. Kaspi, S. et al. Astrophys. J. 533, 631-649 (2000)

9. Zu, Y., Kochanek, C. S. \& Peterson, B. M. Astrophys. J. 735, 80 (2011).

10.Bentz, M. C. \& Katz, S. Publ. Astron. Soc. Pacif. 127, 67-73 (2015)

11. Rakshit, S., Petrov, R. G., Meilland, A. \& Hönig, F. Mon. Not. R. Astron. Soc. 447, 2420-2436 (2015).

\title{
MICROBIOLOGY
}

\section{A bacterium's enemy isn't your friend}

The bacterium Staphylococcus aureus is a leading cause of hard-to-treat human infections. It now seems that, if the bacterium is infected by a virus, a viral enzyme helps the microbe to evade detection by the immune system. SEE LETTER P.705

\section{MICHAEL S. GILMORE \& ONA K. MILLER}

$\mathrm{M}$ icroorganisms thrive on our body's surfaces. The species present are not just a random assembly; rather, they are a community of organisms that are particularly well adapted to the local conditions of temperature, moisture, nutrient availability and host defences ${ }^{1}$. Staphylococcus aureus is one of our most common bacterial residents. It usually lives in nasal, respiratory and reproductive tissues without causing disease, yet, unlike many other resident bacteria, S. aureus has the capacity to give rise to a potentially deadly infection ${ }^{2}$.

During the past 50 years ${ }^{2}$, the resistance of $S$. aureus to antibiotics has become an increasing problem, and strains of the bacterium termed methicillin-resistant $S$. aureus (MRSA), which are resistant to treatment with the antibiotic methicillin and other methylated penicillin-based antibiotics, cause both hospital- and community-acquired infections around the globe. On page 705, Gerlach et al. ${ }^{3}$ describe a previously unknown mechanism whereby viruses influence whether MRSA is recognized by the immune system, shedding light on a process that might tip the balance in determining whether this bacterium will be harmless or disease-causing.

Staphylococcus aureus belongs to the
Gram-positive group of bacteria, and has been described as existing on the borderline between being a normal human microbial resident and a disease-causing organism ${ }^{4}$. This bacterium seems to have the capacity to probe for signs of host weakness, such as reduced immune defences caused by disease. When this is detected, the bacterium can increase its population to a level that can cause the death of the host ${ }^{5}$. Factors that regulate host-microbial interactions are complex, and in addition to host defences, such interactions can be influenced by the presence or absence of other bacteria ${ }^{6}$. Gerlach and colleagues report that viruses can also be part of the mix that influences host-microbial interactions in the context of MRSA.

In Gram-positive bacteria, the cell wall contains polymers known as wall teichoic acids (WTA), which are made up of ribitol phosphate or glycerol phosphate molecules and can constitute up to half of the cellwall mass ${ }^{6}$. Unlike the other main cell-wall component, peptidoglycan, which forms a porous and comparatively insoluble meshwork, WTA form a highly hydrated, gel-like material that fills much of the space between peptidoglycan strands. WTA provide a soluble matrix through which all substances pass before reaching the bacterial cell membrane, and therefore affect bacterial access 
a Strong immune response against WTA

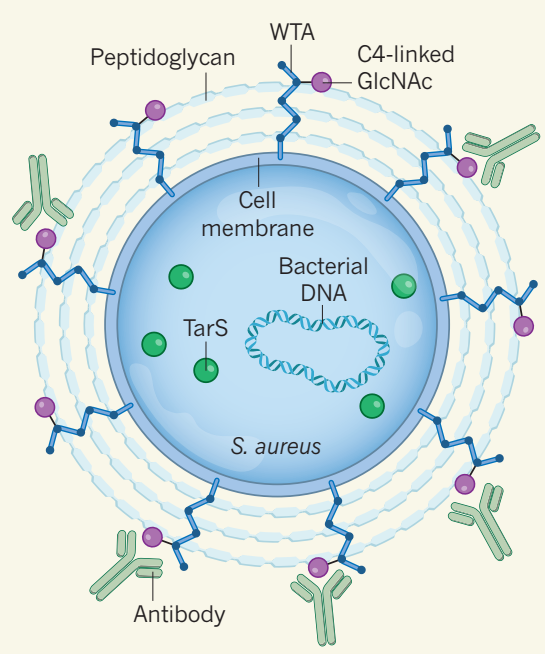

b Weak immune response against WTA

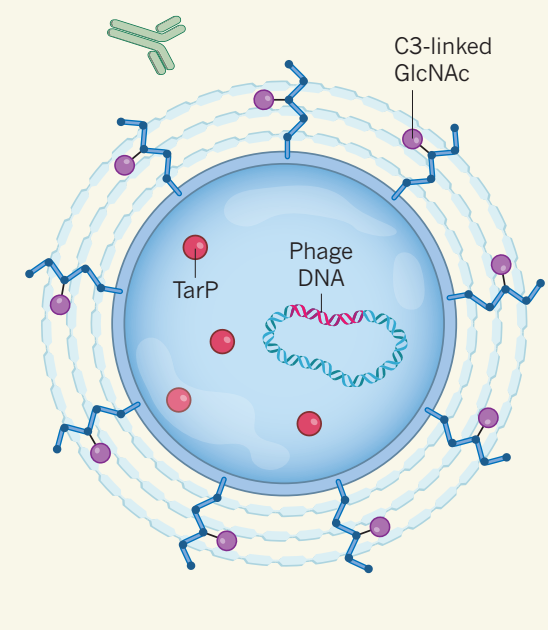

Figure 1 | Viral infection of a bacterium can alter the host's immune response to the microbe. a, The bacterium Staphylococcus aureus is a resident of the human body. Its outer surface is coated with layers of the polymer peptidoglycan crosslinked to wall teichoic acids (WTA) - polymers of ribitol phosphate molecules. The bacterial enzyme TarS modifies WTA, generating a form that has the molecule GlcNAc attached at carbon atoms in the $\mathrm{C} 4$ position in the ribitol. Human antibodies against $S$. aureus often target WTA. b, Gerlach et al. ${ }^{3}$ report that some antibiotic-resistant strains of S. aureus, which are associated with difficult-to-treat infections, have been infected with a virus called a phage. The phage DNA encodes an enzyme called TarP that attaches GlcNAc to WTA at carbon atoms in the $\mathrm{C} 3$ position of ribitol rather than in the normal C4 position. In studies of mice and of human cells, the authors find that these TarP-modified WTA trigger an immune response that is weaker than the response against TarS-modified WTA.

to ions, nutrients, proteins and antibiotics ${ }^{7}$. In S. aureus, WTA are composed of units of D-ribitol phosphate, which are crosslinked to the peptidoglycan (Fig. 1). WTA function is tuned by attachments of the amino acid D-alanine and of $\mathrm{N}$-acetylglucosamine (GlcNAc) ${ }^{7}$ molecules to the ribitol-phosphate polymer.

Gerlach and colleagues decided to investigate whether bacterial evasion of immune-system defences might be one of the reasons that MRSA strains can reach high enough bacterial numbers to cause disease. The authors studied the genome sequences of MRSA strains to identify genes encoding enzymes that modify WTA. This revealed that some MRSA strains encode an enzyme called TarP that catalyses the addition of GlcNAc to $\mathrm{D}$-ribitol phosphate at a particular carbon atom (known as C3) in the ribitol. Normally, GlcNAc is added at a different position, the $\mathrm{C} 4$ carbon, by the action of a related enzyme called TarS.

Surprisingly, the TarP-encoding sequence is of viral origin, and is found in S. aureus as a result of infection by a bacterial virus called a phage. TarP is dominant over its bacterial counterpart, TarS - that is, if both enzymes are present, the GlcNAc linkage is made on the $\mathrm{C} 3$ carbon of ribitol, rather than on the C4 carbon. S. aureus is normally held in check because the immune system has the ability to detect it. However, the authors found that, in mice, the form of WTA made by TarP action is less likely to trigger an immune response than is the form of WTA generated by TarS.

This virus-mediated change to the $S$. aureus cell wall reported by Gerlach and colleagues is important for two reasons. First, it highlights the fact that a fragile truce between host and resident microbe can be affected by the intervention of a third party with its own vested interests. Second, at a time that some ${ }^{8}$ have called the beginning of a 'post-antibiotic era' - given the rise in antibiotic-resistant bacteria and the limited development of new antibiotics reaching the clinic - there is a press-

"A fragile truce
between host
and resident
microbe can be
affected by the
intervention of a
third party."
ing need to develop new strategies to manage infection.

We are now at the dawn of a clinical era in which the goal will be to precisely manage human and microbial interactions to promote health and limit disease. Antibiotics will continue to have a key role, as undoubtedly will other approaches, including the replacement of a person's gut microbes using techniques such as faecal transplants, or the use of phagemediated elimination of undesirable microbes. Determining the best approach will be helped by the development of new diagnostic tools and a clearer understanding of the nature of human and microbial interactions. If deciding whether to take an approach based on a vaccine or possibly using phage treatments in the future, key considerations will include knowing how a bacterium's susceptibility to phage infection varies, and determining whether the presence of phage DNA in a bacterial genome affects the dynamics between human cells and the microbes that colonize the body.

We do not yet know whether the phagemediated alteration of WTA described by Gerlach and colleagues affects where the bacteria reside on the body or the number of bacterial cells present. We also lack a clear understanding of whether the antistaphylococcal WTA-targeting antibodies that most people have, and which do not seem to be protective against infection in immunedeficient individuals, are a 'distraction' imposed by the presence of $S$. aureus. This distraction would keep the immune system busy generating antibodies that end up in ineffective locations such as the bloodstream and do not eliminate the microbe. Alternatively, this low-level immune warfare could represent a stalemate between the host and its resident bacteria.

It is clear that phage-encoded TarP changes the immune reactivity of $S$. aureus. In a model system of human immune cells grown in vitro, the authors found that $S$. aureus strains encoding TarP were cleared from the system less effectively than were $S$. aureus strains that lacked TarP. Similar phage-mediated changes in a bacterial cell surface that alter antibody recognition of the microbe have been reported ${ }^{9}$ for the disease-causing Gramnegative bacterium Shigella flexneri.

Gerlach and colleagues' work, as well as that of others in this area, demonstrates that the balance between host and microbes is a dynamic one. The discovery that phages can have a role in tipping the delicate balance between $S$. aureus colonization and infection might one day affect the choice of approaches for treating MRSA infections.

Michael S. Gilmore and Ona K. Miller are in the Departments of Ophthalmology and of Microbiology and Immunobiology, Harvard Medical School, Massachusetts Eye and Ear Infirmary, Boston, Massachusetts 02114, USA. e-mails:michael_gilmore@meei.harvard.edu; ona_miller@meei.harvard.edu

1. Proctor, D. M. \& Relman, D. A. Cell Host Microbe $\mathbf{2 1}$ 421-432 (2017).

2. Lee, A. S. et al. Nature Rev. Dis. Primers 4, 18033 (2018).

3. Gerlach, D. et al. Nature 563, 705-709 (2018).

4. Camargo, I. L. B. C. \& Gilmore, M. S. J. Bacteriol. 190, 2253-2256 (2008).

5. Guerra, F. E., Borgogna, T. R., Patel, D. M., Sward, E. W. \& Voyich, J. M. Front. Cell Infect. Microbiol. 7, 286 (2017).

6. Boldock, E. et al. Nature Microbiol. 3, 881-890 (2018).

7. Neuhaus, F. C. \& Badilly, J. Microbiol. Mol. Biol. Rev. 67, 686-723 (2003).

8. Zucca, M. \& Savoia, D. Int. J. Biomed. Sci. 6, 77-86 (2010).

9. Mavris, M., Manning, P. A. \& Morona, R. Mol. Microbiol. 26, 939-950 (1997).

This article was published online on 21 November 2018. 